\title{
Gate Leakage Suppression and Breakdown Voltage Enhancement in p-GaN HEMTs using Metal/Graphene Gates
}

\author{
Guangnan Zhou, Zeyu Wan, Gaiying Yang, Yang Jiang, Robert Sokolovskij, Hongyu Yu, and \\ Guangrui (Maggie) Xia
}

\begin{abstract}
In this work, single-layer intrinsic and fluorinated graphene were investigated as gate insertion layers in normallyOFF p-GaN gate HEMTs, which wraps around the bottom of the gate forming Ti/graphene/p-GaN at the bottom and Ti/graphene/ $\mathrm{SiN}_{x}$ on the two sides. Compared to the $\mathrm{Au} / \mathrm{Ti} / \mathrm{p}-\mathrm{GaN}$ HEMTs without graphene, the insertion of graphene can increase the ION/IOFF ratios by a factor of 50 , increase the $V_{T H}$ by $0.30 \mathrm{~V}$ and reduce the off-state gate leakage by 50 times. Additionally, this novel gate structure has better thermal stability. After thermal annealing at $350{ }^{\circ} \mathrm{C}$, gate breakdown voltage holds at $12.1 \mathrm{~V}$, which is first reported for Schottky gate p-GaN HEMTs. This is considered to be a result of the $0.24 \mathrm{eV}$ increase in Schottky barrier height and the better quality of the Ti/graphene/p-GaN and $\mathrm{Ti} /$ graphene/ $\mathrm{SiN}_{x}$ interfaces. This approach is very effective in improving the IoN/IoFf ratio and gate $\mathrm{BV}$ of normally-OFF GaN HEMTs.
\end{abstract}

Index Terms - p-GaN high electron mobility transistor (HEMT), graphene, gate leakage, gate breakdown

\section{INTRODUCTION}

Gallium nitride $(\mathrm{GaN})$ possesses excellent physical properties, such as a high critical electric field and a high saturation velocity [1-3], which is ideal for devices with a low specific ON-resistance $\left(\mathrm{R}_{\mathrm{ON}}\right)$, a high breakdown voltage and a high operation switching frequency. For switch applications, normally-off transistors are required to provide adequate safety conditions [4-5]. Among many options of normally-off HEMTs [6-8], recessed gate metal-insulator-semiconductor-HEMTs (MIS-HEMTs) and p-GaN gate HEMTs (which lift up the conduction band) have been considered as the two most promising approaches [9-10].

Reducing the gate leakage current remains a big challenge for $\mathrm{p}-\mathrm{GaN}$ gate HEMTs [11-13]. The forward gate leakage current limits the gate voltage swing and causes drive losses,

This work was supported by Grant \#2019B010128001 and \#2017A050506002 from Guangdong Science and Technology Department, Grant \#JCYJ20160226192639004 and \#JCYJ20170412153356899 from Shenzhen Municipal Council of Science and Innovation. (Corresponding authors: Y. Hong and G. Xia.)

G. Zhou and G. Xia are with and the School of Microelectronics, Southern University of Science and Technology (SUSTech) and the Department of while the reverse one can lead to the off-state power consumption [14]. Previous studies in MIS-HEMTs revealed that fluorinated graphene can serve as a barrier layer between $\mathrm{Al}_{2} \mathrm{O}_{3}$ and $\mathrm{GaN}$, which contributed to a suppression of gate leakage current by two orders of magnitude [15]. Graphene can act as a strong barrier to atom diffusion and saturate the dangling bonds and defects on the surface $[16,17]$. However, there have not been any reports on the effectiveness of graphene in p-GaN HEMTs, which was addressed in this work.

\section{DEVICE STRUCTURE AND FABRICATION}

The $\mathrm{p}-\mathrm{GaN}$ gate HEMTs were fabricated on $100 \mathrm{~nm}$ $\mathrm{GaN} / 15 \mathrm{~nm} \mathrm{Al}{ }_{0.2} \mathrm{Ga}_{0.8} \mathrm{~N} / 0.7 \mathrm{~nm}$ AlN/4.5 $\mu \mathrm{m} \mathrm{GaN}$ epi-structures grown on $\mathrm{Si}$ (111) substrates by metal organic chemical vapor deposition (MOCVD) provided by Enkris Semiconductor Inc. Fig. 1 shows the schematic cross-section of the devices. The p$\mathrm{GaN}$ layer was doped with $\mathrm{Mg}$ to a concentration of $4 \times 10^{19}$ $\mathrm{cm}^{-3}$. The fabrication flow started with $\mathrm{p}-\mathrm{GaN}$ gate definition by a Cl-based plasma etch followed by a $\mathrm{Cl}_{2} / \mathrm{BCl}_{3}$ plasma etch to form mesas and isolate the devices. The source/drain (S/D) Ohmic contacts were formed by Ti/Al/Ti/Au (20/110/40/50 $\mathrm{nm}$ ) deposition and annealing was at $830{ }^{\circ} \mathrm{C}$ in $\mathrm{N}_{2}$ for $45 \mathrm{s.} 120$ $\mathrm{nm} \mathrm{SiN}_{x}$ deposited by plasma enhanced chemical vapor deposition (PECVD) was used as the first passivation layer. Prior to the deposition of gate metals $(40 \mathrm{~nm} \mathrm{Ti} / 100 \mathrm{~nm} \mathrm{Au})$, single layer graphene grown by chemical vapor deposition (CVD) on $\mathrm{Cu}$ foils was transferred to part of the sample surface via the "polymethyl methacrylate (PMMA)-mediated" wettransfer approach [18]. The undesired part of graphene was etched away by $\mathrm{O}_{2}$ plasma after deposition of gate metal. Finally, the devices were annealed at $350{ }^{\circ} \mathrm{C}$ in $\mathrm{N}_{2}$ for 5 minutes to improve the $\mathrm{Au} / \mathrm{Ti}$ (graphene)/p-GaN interface.

Materials Engineering, the Univerisity of British Columbia (UBC). (e-mail: gxia@mail.ubc.ca)

Z. Wan, Y. Jiang, R. Sokolovskij and H. Yu are with the School of Microelectronics, SUSTech; GaN Device Engineering Technology Research Center of Guangdong, SUSTech; and the Key Laboratory of the Third Generation Semiconductors, SUSTech, 518055 Shenzhen, Guangdong, China. (e-mail: yuhy@ sustech.edu.cn)

G. Yang is with the School of Innovation \& Entrepreneurship at SUSTech. 


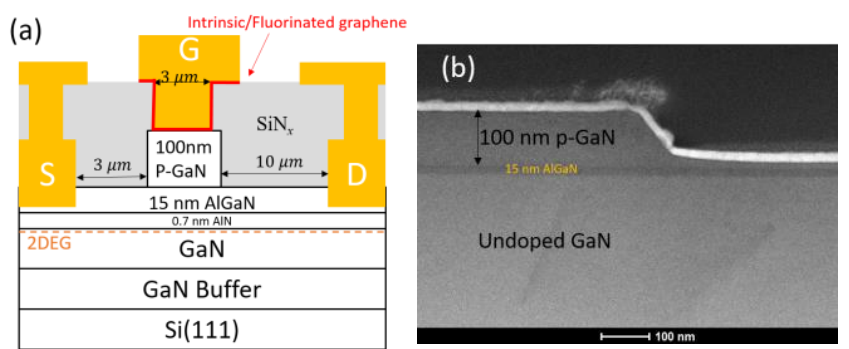

Fig. 1. (a) Schematic of the device structure; (b) Cross-section of the device characterized by a scanning transmission electron microscope (STEM) showing the edge of a $\mathrm{p}-\mathrm{GaN}$ gate and the $\mathrm{p}-\mathrm{GaN} / \mathrm{AlGaN} / \mathrm{GaN}$ structure.

Two types of graphene, intrinsic (I-graphene) and fluorinated graphene (F-graphene), were investigated. Fgraphene was realized by exposing the I-graphene to $\mathrm{SF}_{6}$ plasma. In this experiment, the quality and the cleanness of the transferred graphene are crucial, as the metal/semiconductor barrier height heavily depends on the surface states and defects. Especially, the PMMA residuals could lead to Fermi-level pinning at the metal/graphene/p-GaN junction, which would eventually result in the malfunction of these devices [19]. As illustrated in Fig. 2, the Raman spectra and SEM pictures show that the transferred graphene has a high quality $\left(I_{2 D} / I_{G} \approx 2\right)$ with negligible PMMA contaminations [20].
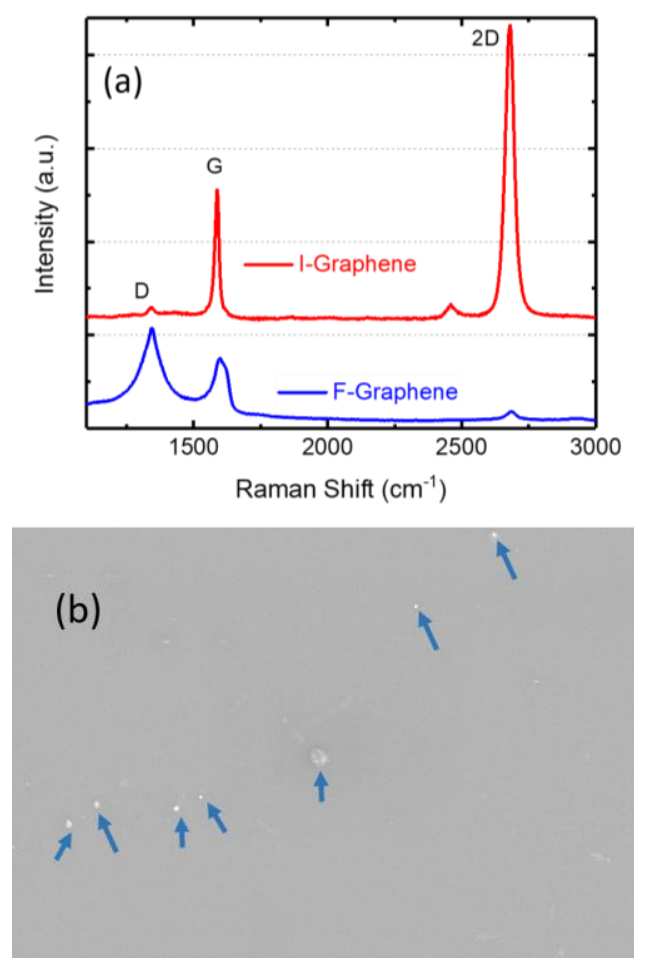

Fig. 2. The transferred graphene characterized by (a) Raman spectroscopy and (b) SEM, a few PMMA residuals are shown intentionally as noted by the arrows.

\section{RESULTS AND DISCUSSION}

\section{A. HEMTs results}

The HEMTs with and without graphene are from the same wafer piece. The gate leakage, transfer and output characteristics of the HEMTs are shown in Fig. 3.

Compared to the HEMTs with Ti/p-GaN gate structures, those with Ti/I-graphene/p-GaN and Ti/F-graphene/p-GaN gate structures have about one order of magnitude lower gate leakage with a forward bias and 2 orders of magnitude with a reverse bias.
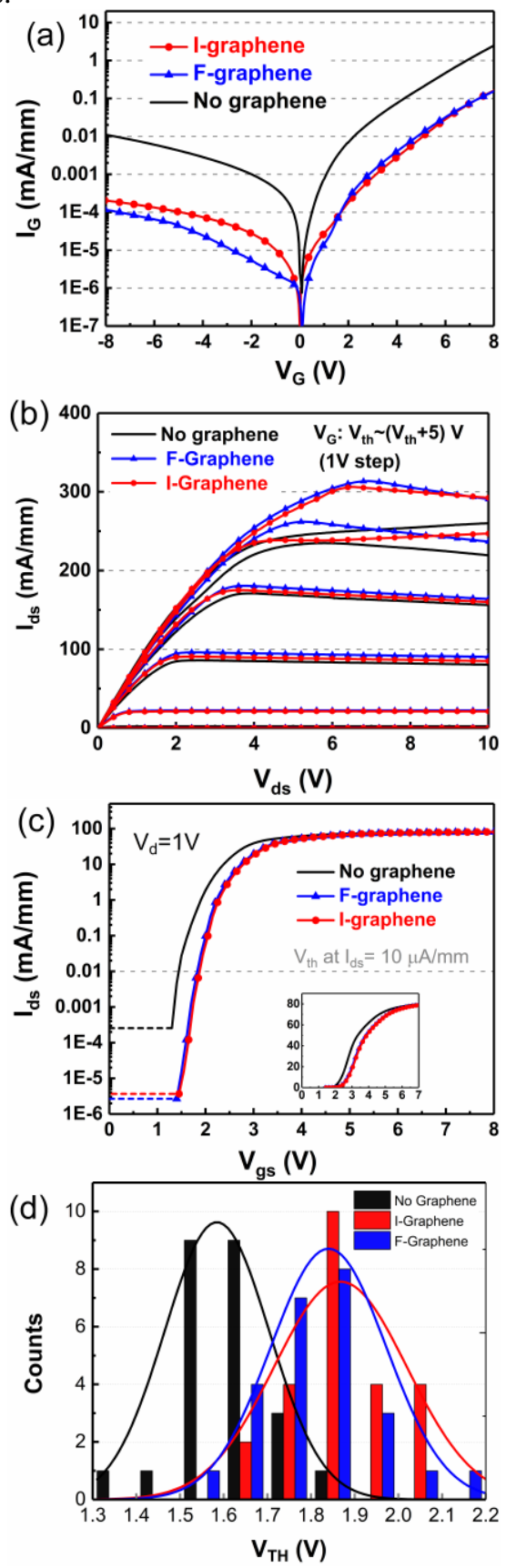

Fig. 3. Comparison of the device performance of the p-GaN HEMTs with Igraphene, F-graphene and without graphene; (a) gate leakage characteristics under $\mathrm{V}_{\mathrm{DS}}=0 \mathrm{~V}$; (b) output characteristics $\left(\mathrm{V}_{\mathrm{G}}\right.$ from $\mathrm{V}_{\mathrm{TH}}$ to $\left.\left(\mathrm{V}_{\mathrm{TH}}+5 \mathrm{~V}\right)\right), 1 \mathrm{~V}$ step); (c) transfer characteristics under $\mathrm{V}_{\mathrm{DS}}=1 \mathrm{~V}$, the inset figure is transfer characteristics plotted in linear scale; and (d) $\mathrm{V}_{\mathrm{TH}}$ uniformity characterization.

Additionally, the HEMTs with graphene have higher threshold voltage $\left(\mathrm{V}_{\mathrm{TH}}\right)$ defined at $\mathrm{I}_{\mathrm{DS}}=10 \mu \mathrm{A} / \mathrm{mm}(0.32 \mathrm{~V}$ higher for I-graphene and $0.28 \mathrm{~V}$ higher for F-graphene). More importantly, the insertion of graphene increased the $\mathrm{I}_{\mathrm{ON}} / \mathrm{I}_{\mathrm{OFF}}$ ratio at least one order of magnitude for both types of graphene. Detailed comparison of the extracted DC parameters between three types of devices is shown in Table I. The statistical data of $V_{T H}$ is shown in Fig. 3 (d). The average $V_{T H}$ values of the 
devices without graphene, with I-graphene and with F-graphene are $1.58 \mathrm{~V}, 1.86 \mathrm{~V}$ and $1.84 \mathrm{~V}$, respectively. The increase of $\mathrm{V}_{\text {TH }}$ should be attributed to higher Schottky barrier height $\left(\Phi_{B}\right)$ and p-type doping in graphene introduced in the "PMMAmediated" wet-transfer process [18]. In summary, the insertion of graphene reduced the gate leakage current and increased $\mathrm{V}_{\mathrm{TH}}$ without sacrificing any output performances.

TABLE I

COMPARISON OF EXTRACTED DC PARAMETERS

\begin{tabular}{cccc}
\hline \hline Parameters & No graphene & I-Graphene & F-Graphene \\
\hline $\mathrm{I}_{\mathrm{G}}\left(\mathrm{V}_{\mathrm{G}}=8 \mathrm{~V}\right)$ & $\sim 2$ & $\sim 0.1$ & $\sim 0.1$ \\
$(\mathrm{~mA} / \mathrm{mm})$ & & $3.6 \times 10^{-6}$ & $1.2 \times 10^{-6}$ \\
$\mathrm{I}_{\mathrm{OFF}}(\mathrm{mA} / \mathrm{mm})$ & $2.5 \times 10^{-4}$ & 1.86 & 1.82 \\
$\mathrm{~V}_{\mathrm{TH}}(\mathrm{V})$ & 1.54 & 12.8 & 11.9 \\
$\mathrm{R}_{\mathrm{ON}}(\Omega \cdot \mathrm{mm})$ & 12.4 & 12.05 & 12.0 \\
Gate $\mathrm{BV}(\mathrm{V})$ & 9.8 &
\end{tabular}

\section{B. Effects of Graphene during Annealing Treatment}

In practice, passivation layers are necessary for metal pads and interconnect protection. This means that the gate metal/p$\mathrm{GaN}$ interfaces will commonly experience some lowtemperature thermal annealing. For the gate-metal-first process, the interface will undergo an even higher thermal budget for S/D contact annealing [13]. To investigate the effects of graphene during these thermal steps, the gate leakage of devices had also been measured before the annealing treatment $\left(350{ }^{\circ} \mathrm{C}\right.$ in $\mathrm{N}_{2}$ for 5 minutes). Fig. 4 presents the gate leakage characteristics and gate breakdown voltage (BV) before and after annealing.
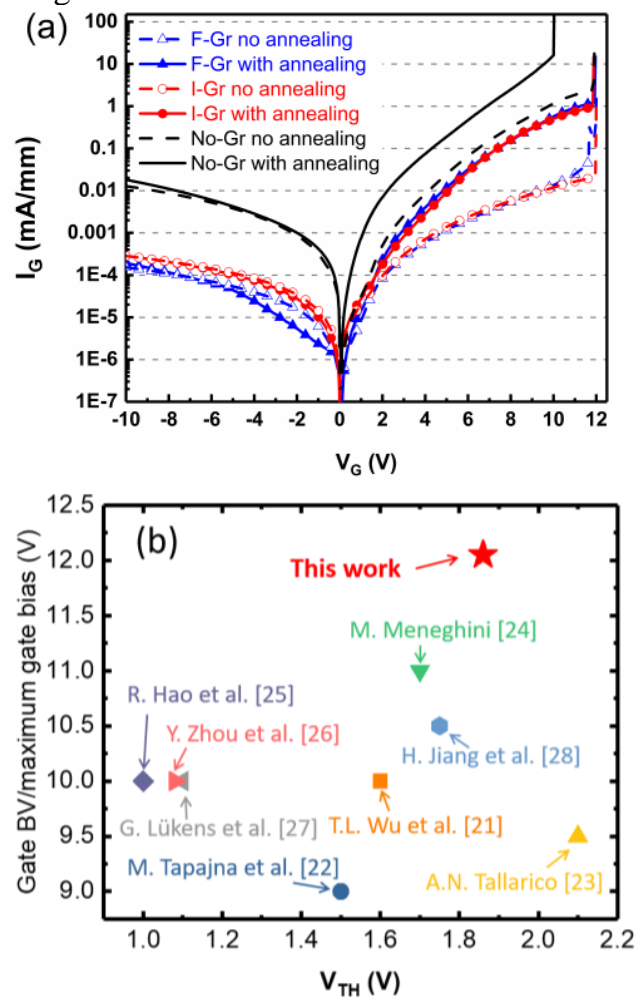

Fig. 4. (a) Comparison of the gate leakage current and gate breakdown voltage before and after $350{ }^{\circ} \mathrm{C} 5 \mathrm{~min}$ annealing; (b) Gate BV or maximum gate bias versus threshold voltage for $\mathrm{p}-\mathrm{GaN}$ gate HEMTs fabricated by several groups.

As illustrated in Fig. 4, in the case of forward $V_{G}$ bias, all the devices have a considerable increase in gate leakage. This should be due to the reduction of $\Phi_{B}$. After the annealing, the gate $\mathrm{BV}$ of the devices without graphene was $9.80 \mathrm{~V}$ whereas the devices with graphene broke down at $12.05 \mathrm{~V}$. To our knowledge, this is the highest gate $\mathrm{BV}$ that has been reported in HEMTs with p-GaN Schottky gates. Fig. 7 summarize the gate $\mathrm{BV}$ or maximum gate bias versus $\mathrm{V}_{\mathrm{TH}}$ for $\mathrm{p}-\mathrm{GaN}$ gate HEMTs fabricated by several groups. Compared with other reported pGaN gate HEMTs, our devices with graphene insertion exhibit the highest gate $\mathrm{BV}$ and a relative high $\mathrm{V}_{\mathrm{TH}}$. These improvements are crucial for $\mathrm{p}-\mathrm{GaN}$ gate HEMT device for power switching applications.

To get further insights into the role of graphene on Ti/p-GaN interfaces, scanning transmission electron microscopy (STEM) images of the HEMTs were obtained. As shown in Fig. 5, the $\mathrm{Ti} / \mathrm{p}-\mathrm{GaN}$ interface quality has been much improved by the insertion of graphene. The thin darker regions pointed out by the yellow arrows in Fig. 5(a) were confirmed to be small voids or TiN islands by energy dispersive spectroscopy (EDS), as illustrated in the insets of Fig. 5(a). The inset figure on the left shows that the darker region has TiN, while the right one illustrates a void where only background signals/noise exist in EDS. Meanwhile, the interface with graphene is more uniform and cleaner. This means that the graphene interlayer helped to reduce the formation of TiN and interface voids and maintain a more stable Ti/graphene/p-GaN interface during annealing, contributing to a lower leakage current and a higher gate BV.

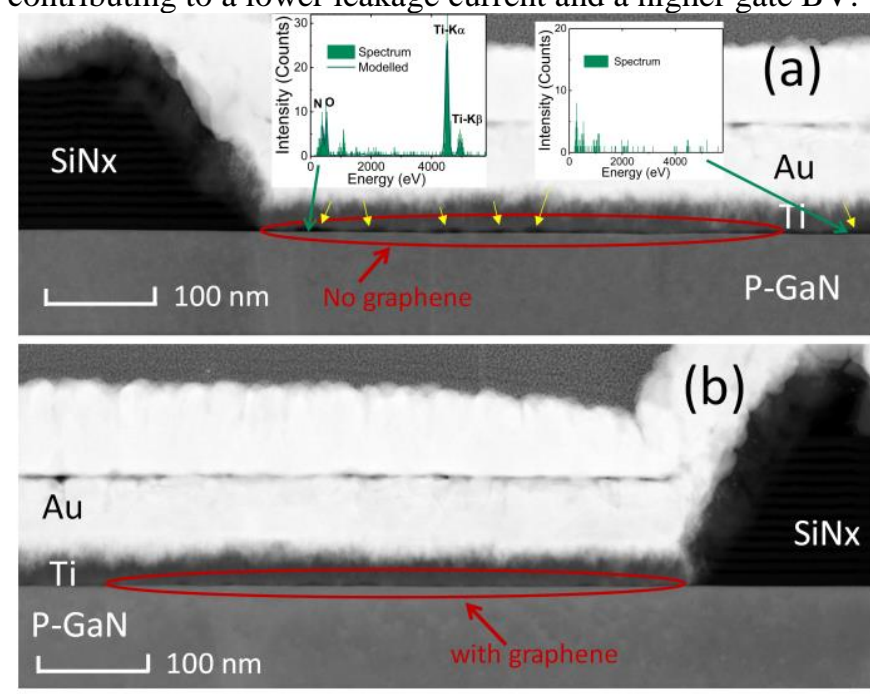

Fig. 5. Cross-section STEM of the HEMTs after $350{ }^{\circ} \mathrm{C} 5 \mathrm{~min}$ annealing in the gate region (a) without graphene; Insets: EDS show the darker regions are either TiN or voids; (b) with I-graphene. The Au/Ti/p-GaN interface has been much improved by graphene.

\section{Mechanism Study with Ti/graphene/p-GaN Schottky diodes}

Besides the material analysis, Au/Ti/graphene/p-GaN Schottky contacts have been fabricated as seen in the inset of Fig. 6(a). Considering the fact that the I-graphene and Fgraphene had similar effects in the previous experiments, only I-graphene was studied here. This test structure consists of two Schottky contacts back-to-back [29]. The I-V measurements were carried out in the bias range of 0 to $6 \mathrm{~V}$. Before annealing, 
the systems with and without I-graphene have comparable current density, whereas they are significantly different after $350{ }^{\circ} \mathrm{C} 5$ minutes annealing.

The temperature dependence of the I-V characteristics has also been studied for these systems to extract $\Phi_{B}$. Taking the $\mathrm{Mg}$ doping concentration of $4 \times 10^{19} \mathrm{~cm}^{-3}$ into consideration, the value of $E_{00} / k T$ is in the range between 0.85 and 1.34 in the examined temperature range. This implies that thermionic field emission (TFE) should be the dominant mechanism in our systems. Hence, the expression of the TFE reverse current was used to fit our experimental data $[13,30]$

$$
\begin{aligned}
I_{s}= & \frac{A_{c} A^{*} T \sqrt{\pi E_{00}}}{k} \cdot \sqrt{q\left(V-V_{n}\right)+\frac{q \Phi_{B}}{\cosh ^{2}\left(\frac{E_{00}}{k T}\right)}} \\
& \cdot \exp \left(-\frac{q \Phi_{B}}{E_{00} \operatorname{coth}\left(\frac{E_{00}}{k T}\right)}\right) \\
I_{T F E}= & I_{S} \cdot \exp \left(\frac{q V}{k T}-\frac{q V}{E_{00} \operatorname{coth}\left(\frac{E_{00}}{k T}\right)}\right) \\
E_{00}= & \frac{q h}{4 \pi} \sqrt{\frac{N_{A}}{m^{*} \varepsilon}}
\end{aligned}
$$

where $V$ is the applied voltage, $A_{c}$ is the contact area, $A^{*}$ is the Richardson constant, $q$ is the elementary charge, $k$ is the Boltzmann constant, $h$ is Planck's constant, $T$ is the absolute temperature, and $\varepsilon$ and $m^{*}$ are the dielectric constant for GaN and the effective mass for holes, respectively. $\Phi_{B}$ represents the Schottky barrier height of the metal(graphene)/p-GaN interface. In our calculation, we used the values of $\varepsilon=8.9 \varepsilon_{0}, A^{*}=$ $27.9 \mathrm{~A} / \mathrm{cm}^{2} \mathrm{~K}^{2}$, and $\mathrm{m}^{*}=0.81 \mathrm{~m}_{0}$.
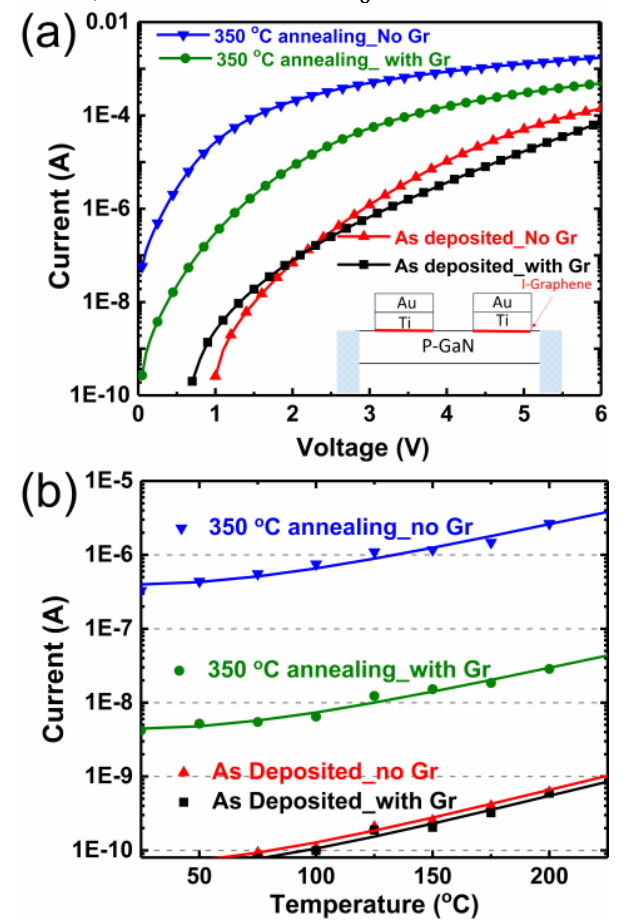

Fig. 6. (a) $\mathrm{I}-\mathrm{V}$ characteristics acquired in back-to-back diodes. Inset: Schematics of the sample structure; (b) Temperature dependence of the current measured at $\mathrm{V}=+0.2 \mathrm{~V}$ in back-to-back Schottky contacts. The symbols are measured data. The lines are the fitting curves.

The extracted $\Phi_{B}$ from the data (Fig. 6(b)) is $2.08 \mathrm{eV}$ for the contacts without graphene and $2.09 \mathrm{eV}$ for those with Igraphene, respectively. After annealing the sample at $350{ }^{\circ} \mathrm{C}$ in $\mathrm{N}_{2}$ for $5 \mathrm{~min}, \Phi_{B}$ decreased to $1.65 \mathrm{eV}$ (no Gr) and $1.89 \mathrm{eV}$ (with graphene), which results in approximately one order of magnitude smaller current in the sample with graphene. The extracted $\Phi_{B}$ are summarized in Table II. These data are consistent with the experimental value reported in the literature for Ti/p-GaN interfaces [13].

It is worth mentioning that this back-to-back Schottky diodes experiment was conducted on $\mathrm{p}-\mathrm{GaN}$ wafer without any passivation or etching process. The metal $/ \mathrm{p}-\mathrm{GaN}$ interfaces in a HEMT can be more defective due to the ion bombardment during the fabrication process (e.g. passivation deposition and gate dielectric etching).

\section{Analysis and Modeling of Gate Leakage Current}

In this part, the gate current characteristics in Fig. 4(a) and the corresponding mechanism will be analyzed more thoroughly. The data will be fitted to a physical model from Ref. [31]. The trap depth and the corresponding prefactors of the HEMTs with/without graphene will be extracted.

As shown in Fig. 7, the gate structure includes two junctions, i.e. the metal (graphene)/p-GaN junction (Schottky junction) and the $\mathrm{p}-\mathrm{GaN} / \mathrm{AlGaN} / \mathrm{GaN}$ junction (p-i-n junction). The gate leakage mechanisms are different in different gate bias regimes.

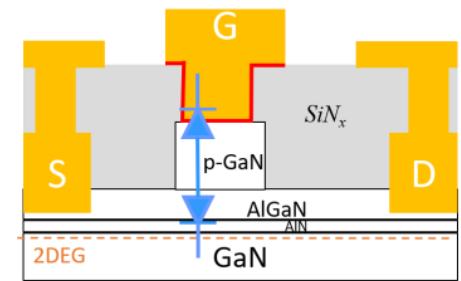

Fig. 7. Schematic representation of the Au/Ti/p-GaN/AlGaN/GaN HEMT and its equivalent circuit.

In the $\mathrm{V}_{\mathrm{G}}<0$ regime, vertical gate current is blocked due to the reverse bias of the p-i-n junction. An electron current is likely from the Schottky metal near the gate edges through a leakage path along the $\mathrm{p}-\mathrm{GaN}$ sidewall and these electrons are emitted into the 2DEG layer. As shown in Fig. 4(a), the $I_{G}$ with/without graphene at reverse bias had negligible change after annealing even though the Schottky barrier changed. This further confirms that the dominant factor for $\mathrm{I}_{\mathrm{G}}$ at the negative bias is associated with the surface defects caused during the fabrication process. A similar conclusion has also been reported for previous p-GaN gate HEMTs [31, 32]. According to Ref [31, 32], the dominant gate leakage mechanism associated with pGaN surface defects is found to be Poole-Frenkle emission (PFE) for the case of $V_{G}<0$ as shown in Fig. 7(b), which can be described by:

$$
J=C E \exp \left(-\frac{q\left(\Phi_{t}-\beta \sqrt{E}\right)}{k T}\right),
$$

where $C=q n \mu_{n}, \beta=\sqrt{q / \pi \epsilon}$ is the Schottky factor, $k$ is Boltzmann's constant, $q \Phi_{t}$ is the trap depth, and $E=V_{G S} / L_{G S}$ is the electric field, with $L_{G S}$ the shortest distance between the gate and source metal.

The temperature dependent $\mathrm{I}_{\mathrm{G}}-\mathrm{V}_{\mathrm{G}}$ of the HEMTs after 
annealing have been measured from $300 \mathrm{~K}$ to $450 \mathrm{~K}$. The PFE model has been used to fit the data in the negative bias, with the trap depth $q \Phi_{t}$ and prefactor $\mathrm{C}$ as the fitting parameters (Fig. 8). The fitting results are illustrated in Fig. 8, where the fitting curves are plotted as solid lines and symbols represent selected measurement points. The trap depths are $q \Phi_{t, I-G r}=$ $0.199 \mathrm{eV}$ and $q \Phi_{t, n o-G r}=0.165 \mathrm{eV}$, respectively. The prefactor $\mathrm{C}$ in the HEMTs without graphene is $\frac{C_{n o-G r}}{C_{I-G r}}=$ $\frac{585.70}{47.46}=12.34$ times of that with I-graphene. This explains why $\mathrm{I}_{\mathrm{G}}$ in the HEMTs with/without graphene differ a lot in the asdeposited case even though their $\Phi_{B}$ are comparable extracted in Section II-C. Graphene layers passivated the p-GaN surface resulting in the neutralization of interface states and, consequently, less electron leakage toward the gate foot along the $\mathrm{p}-\mathrm{GaN}$ surface.

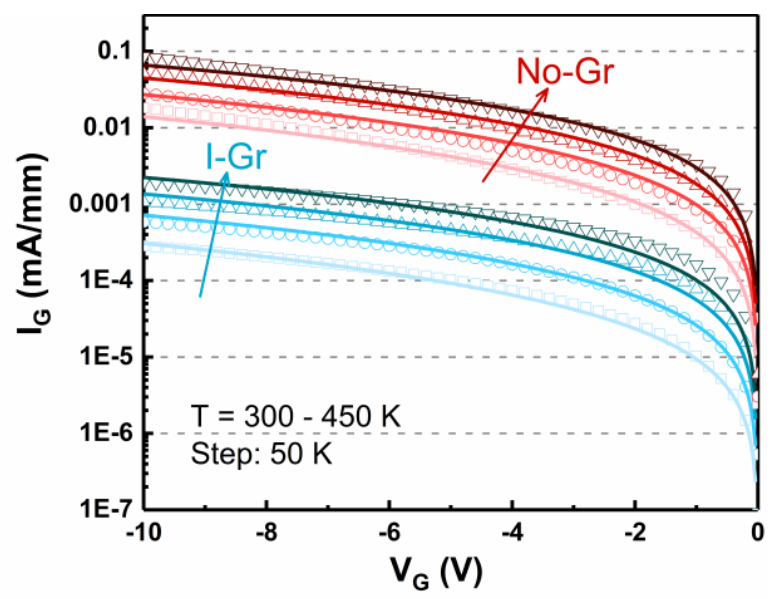

Fig. 8. Negative gate bias current from $\mathrm{T}=300$ to $450 \mathrm{~K}$ measured on HEMTs with I-graphene and without graphene. The symbols are selected measured data. The lines are the fitting curves using the PFE model.

TABLE II

SUMMARY OF MODELLING RESULTS OF THE BACK-TO-BACK DIODES AND THE HEMTS GATE CURRENT

\begin{tabular}{cccc}
\hline \hline & As Deposited & $\begin{array}{c}\mathbf{3 5 0}^{\circ} \mathbf{C} \text { 5 min } \\
\text { annealing }\end{array}$ \\
\hline$\Phi_{B}(\mathrm{eV})$ in & With I-graphene & 2.08 & 1.89 \\
diodes & No graphene & 2.09 & 1.65 \\
\hline$\Phi_{t}(\mathrm{eV})$ in & With I-graphene & $\backslash$ & 0.199 \\
HEMTs & No graphene & 1 & 0.165 \\
\hline$C\left(\Omega^{-1}\right)$ in & With I-graphene & $\backslash$ & $5.86 \times 10^{-7}$ \\
HEMTs & No graphene & 1 & $4.75 \times 10^{-8}$ \\
\hline \hline
\end{tabular}

In the $\mathrm{V}_{\mathrm{G}}>0$ regime, the $\mathrm{p}$-i-n junction will be positive bias while the Schottky junction will be reverse bias. In the case of no-annealing, it is found that the $\mathrm{I}_{\mathrm{G}}$ are comparable in the regime of positive and negative gate bias in Fig 4(a). We deduce that the vertical gate current is also small since the Schottky barrier is large enough. The graphene contributes to a lower gate leakage by reducing the interface defects similar to the $\mathrm{V}_{\mathrm{G}}$ $<0$ regime. In the case of after-annealing, the gate leakage current in positive bias is much larger than in negative bias. The dominant current shall be from the vertical gate current due to a lower $\Phi_{B}$ in the Schottky junction. The voltage dropped at the $\mathrm{p}$-i-n junction is large enough to turn on the diode and the dominant conduction mechanism is found to be thermionic field emission. Thus, graphene helps to reduce the gate leakage current by maintaining a more stable interface and a higher $\Phi_{B}$ after annealing as analyzed in Section II-C.

Based on these evidences, it can be deduced that graphene can help reduce the interface defects and maintain a more stable $\mathrm{Au} / \mathrm{Ti} / \mathrm{p}-\mathrm{GaN}$ interface during annealing. In $\mathrm{V}_{\mathrm{G}}>0$ regime, the larger $\Phi_{B}$ contributes to a lower gate leakage current and the higher $\mathrm{V}_{\mathrm{TH}}$. Graphene can also prevent the decrease of gate $\mathrm{BV}$ during annealing. In the $\mathrm{V}_{\mathrm{G}}<0$ regime, as the defects at the $\mathrm{p}$ $\mathrm{GaN}$ surfaces during the fabrication process are the dominant factor for gate leakage, graphene reduces the gate leakage by saturating the defects at the $\mathrm{p}-\mathrm{GaN}$ surface. According to fitting the experimental data, it is found that graphene changes the trap depth $q \Phi_{t}$ from $0.165 \mathrm{eV}$ to $0.199 \mathrm{eV}$ and reduces the prefactor $\mathrm{C}$ by 12.3 times.

\section{CONCLUSION}

In this work, intrinsic and fluorinated graphene were investigated as gate insertion layers for normally-OFF p-GaN HEMTs, which formed Au/Ti/graphene/p-GaN interfaces in the middle and $\mathrm{Au} / \mathrm{Ti} / \mathrm{graphene} / \mathrm{SiN}_{x}$ on the two sides. 50 times larger $\mathrm{I}_{\mathrm{ON}} / \mathrm{I}_{\mathrm{OFF}}$ ratios, $0.30 \mathrm{~V}$ higher $\mathrm{V}_{\mathrm{TH}}$ increase, 50 times offstate gate leakage reduction have been achieved by the insertion of graphene. For the first time, $12.1 \mathrm{~V}$ gate $\mathrm{BV}$ has been achieved with I-graphene for Schottky gate p-GaN HEMTs. This is considered to be the result of a $0.24 \mathrm{eV}$ higher $\Phi_{B}$, and the better graphene/p-GaN interfaces. In the negative gate bias, $\mathrm{I}_{\mathrm{G}}$ has been fitted to PFE model, which revealed that graphene layers contribute to a $0.034 \mathrm{eV}$ larger trap depth and a 12.3-time smaller prefactor. As single-layer graphene can be prepared in wafer-size areas [33], This approach is mass-production compatible and very effective in improving the $\mathrm{I}_{\mathrm{ON}} / \mathrm{I}_{\mathrm{OFF}}$ ratios and increasing $\mathrm{V}_{\mathrm{TH}}$ and gate $\mathrm{BV}$ of $\mathrm{p}-\mathrm{GaN}$ gate HEMTs.

\section{ACKNOWLEDGMENT}

The work was conducted at Materials Characterization and Preparation Center (MCPC) at SUSTech, and we acknowledge the technical support from the staff and engineers at MCPC. Dr. Mengyuan Hua from SUSTech is acknowledged for useful discussions.

\section{REFERENCES}

[1] F. Ren and J. C. Zolper, Wide Energy Bandgap Electronic Devices. Singapore: World Scientific, 2003.

[2] F. Roccaforte et al., "Recent advances on dielectrics technology for $\mathrm{SiC}$ and GaN power devices," Appl. Surf. Sci., vol. 301, pp. 9-18, May 2014.

[3] F. Roccaforte et al., "Challenges for energy efficient wide band gap semiconductor power devices," Phys. Status Solidi A, vol. 211, no. 9, pp. 2063-2071, Sep. 2014.

[4] M. Su, C. Chen, and S. Rajan, "Prospects for the application of GaN power devices in hybrid electric vehicle drive systems," Semicond. Sci. Technol., vol. 28, no. 7, p. 074012, 2013.

[5] M. J. Scott et al., "Merits of gallium nitride based power conversion," Semicond. Sci. Technol., vol. 28, Jul. 2013, Art. no. 074013.

[6] Y. Cai, Y. Zhou, K. J. Chen, and K. M. Lau, IEEE Electron Device Lett. 26, 435 (2005).

[7] Y. Uemoto, M. Hikita, H. Ueno, H. Matsuo, H. Ishida, and M. Yanagihara, IEEE Trans. Electron Devices 54, 3393 (2007). 
[8] H. Y. Wang, J. Y. Wang, J. Q. Liu, M. J. Li, Y. D. He, M. J. Wang, M. Yu, W. G. Wu, Y. Zhou, and G. Dai, Appl. Phys. Express 10, 106502 (2017).

[9] G. Greco, F. Iucolano, and F. Roccaforte, "Review of technology for normally-off HEMTs with p-GaN gate," Mater. Sci. Semicond. Process., vol. 78, no. September 2017, pp. 96-106, 2018.

[10] E. A. Jones, F. F. Wang, and D. Costinett, IEEE J. Emerging Sel. Top. Power Electron. 4, 707 (2016).

[11] A. Stockman et al., "On the origin of the leakage current in p-gate AlGaN/GaN HEMTs," IEEE Int. Reliab. Phys. Symp. Proc., vol. 2018March, p. 4B.51-4B.54, 2018.

[12] N. Xu et al., "Gate leakage mechanisms in normally-off pGaN/AlGaN/GaN high electron mobility transistors," Appl. Phys. Lett., vol. 113, no. 15, 2018.

[13] G. Greco, F. Iucolano, S. Di Franco, C. Bongiorno, A. Patti, and F. Roccaforte, "Effects of Annealing Treatments on the Properties of $\mathrm{Al} / \mathrm{Ti} / \mathrm{p}-\mathrm{GaN}$ Interfaces for Normally-off p-GaN HEMTs," IEEE Trans. Electron Devices, vol. 63, no. 7, pp. 2735-2741, 2016.

[14] M. H. Mi, X. H. Ma, L. Yang, H. Bin, J. J. Zhu, Y. L. He, M. Zhang, S. Wu, and Y. Hao, Appl. Phys. Lett. 111, 173502 (2017).

[15] L. Shen, D. Zhang, X. Cheng, et al., "Performance Improvement and Current Collapse Suppression of $\mathrm{Al}_{2} \mathrm{O}_{3} / \mathrm{AlGaN} / \mathrm{GaN}$ HEMTs Achieved by Fluorinated Graphene Passivation," IEEE Electron Device Lett., vol. 38, no. 5, pp. 596-599, 2017.

[16] S. C. O’Hern, C. A. Stewart, M. S. H. Boutilier, J.-C. Idrobo, S. Bhaviripudi, S. K. Das, J. Kong, T. Laoui, M. Atieh, and R. Karnik, "Selective molecular transport through intrinsic defects in a single layer of CVD graphene," ACS Nano, vol. 6, no. 11, pp. 10130-10138, 2012, doi: $10.1021 / \mathrm{nn} 303869 \mathrm{~m}$.

[17] S. Hu, M. Lozada-Hidalgo, F. C. Wang, A. Mishchenko, F. Schedin, R. R. Nair, E. W. Hill, D. W. Boukhvalov, M. I. Katsnelson, R. A. Dryfe, I. V. Grigorieva, H. A. K. Wu, and A. Geim, "Proton transport through one-atom-thick crystals," Nature, vol. 516, no. 11, pp. 227-230, Dec. 2014, doi: 10.1038/nature14015.

[18] Liang X, Sperling B A, Calizo I, et al. Toward clean and crackless transfer of graphene[J]. ACS nano, 2011, 5(11): 9144-9153.

[19] Giubileo F, Di Bartolomeo A. The role of contact resistance in graphene field-effect devices[J]. Progress in Surface Science, 2017, 92(3): 143175 .

[20] Ferrari A C, Basko D M. Raman spectroscopy as a versatile tool for studying the properties of graphene[J]. Nature nanotechnology, 2013, 8(4): 235

[21] T. L. Wu et al., "Forward Bias Gate Breakdown Mechanism in Enhancement-Mode p-GaN Gate AlGaN/GaN High-Electron Mobility Transistors," IEEE Electron Device Lett., vol. 36, no. 10, pp. 1001-1003, 2015.

[22] M. Ťapajna, O. Hilt, E. Bahat-Treidel, J. Würfl, and J. Kuzmik, "Gate reliability investigation in normally-off p-type-GaN Cap/AlGaN/GaN HEMTs under forward bias stress," IEEE Electron Device Lett., vol. 37, no. 4, pp. 385-388, 2016.

[23] A. N. Tallarico et al., "Investigation of the p-GaN gate breakdown in forward-biased GaN-based power HEMTs," IEEE Electron Device Lett., vol. 38, no. 1, pp. 99-102, 2017.

[24] M. Meneghini et al., "Gate stability of GaN-Based HEMTs with P-Type Gate," Electron., vol. 5, no. 2, pp. 1-8, 2016.

[25] R. Hao et al., "Normally-off p -GaN / AlGaN / GaN high electron mobility transistors using hydrogen plasma treatment," vol. 152106, no. 2016, pp. 1-5, 2017.

[26] Y. Zhou et al., "P-GaN Gate Enhancement-Mode HEMT Through a High Tolerance Self-Terminated Etching Process," IEEE J. Electron Devices Soc., vol. 5, no. 5, pp. 340-346, 2017.

[27] G. Lukens, H. Hahn, H. Kalisch, and A. Vescan, "Self-Aligned Process for Selectively Etched p-GaN-Gated AlGaN/GaN-on-Si HFETs," IEEE Trans. Electron Devices, vol. 65, no. 9, pp. 3732-3738, 2018.

[28] H. Jiang, R. Zhu, Q. Lyu, and K. M. Lau, "High-Voltage p-GaN HEMTs with OFF-State Blocking Capability after Gate Breakdown," IEEE Electron Device Lett., vol. 40, no. 4, pp. 530-533, 2019.

[29] D. A. Neamen, Electronic Circuit Analysis and Design, 2nd ed. New York, NY, USA: McGraw-Hill, 2002.

[30] N. Miura et al., "Thermal annealing effects on Ni/Au based Schottky contacts on $\mathrm{n}-\mathrm{GaN}$ and $\mathrm{AlGaN} / \mathrm{GaN}$ with insertion of high work function metal," Solid-State Electron., vol. 48, no. 5, pp. 689-695, May 2004.

[31] A. Stockman et al., "Gate Conduction Mechanisms and Lifetime Modeling of p-Gate AlGaN/GaN High-Electron-Mobility Transistors," IEEE Trans. Electron Devices, vol. 65, no. 12, pp. 5365-5372, 2018.
[32] N. Xu et al., "Gate leakage mechanisms in normally off pGaN/AlGaN/GaN high electron mobility transistors," Appl. Phys. Lett., vol. 113, no. 15, 2018.

[33] S. Bae, H. Kim, Y. Lee, X. Xu, et al. "Roll-to-roll production of 30-inch graphene films for transparent electrodes." Nature nanotechnology 5.8 (2010): 574 . 